\title{
El olvido de la demografía en los estudios de Sostenibilidad
}

\author{
Daniel Gil Pérez y Amparo Vilches \\ Departament de Didàctica de les Ciències Experimentals i Socials. Facultat de Magisteri. \\ Universitat de València. AvdaTarongers 4. Valencia . España.
}

[Recibido el 29 de septiembre de 2017, aceptado el 21 de noviembre de 2017]

\begin{abstract}
A lo largo de la historia del Homo sapiens, su número se ha mantenido muy reducido y hubo que esperar a principios del siglo XIX para que alcanzara los mil millones. Se inició entonces un rápido crecimiento y durante el siglo XX la población mundial casi se cuadriplicó, superando los 6000 millones, lo que llevó a estudiar, con lógica preocupación, los posibles efectos de esta explosión demográfica sobre el conjunto de la biosfera y sobre la propia especie humana. Hoy, sin embargo, cuando se han sobrepasado los 7500 millones y la población mundial sigue aumentando en unos 80 millones cada año, la problemática demográfica ha dejado de tener una presencia relevante en los estudios y publicaciones sobre los problemas que caracterizan la grave situación de emergencia planetaria y la necesidad de una urgente transición a sociedades sostenibles, a la que debe contribuir la educación científica. En este trabajo intentamos profundizar en las razones de esta falta de atención a la demografía, estudiando la validez de los argumentos que suelen esgrimirse para justificar la ausencia de la problemática demográfica en la consideración de los límites del planeta y, a partir de este estudio, extraer las lecciones pertinentes para mejor orientar la necesaria transición a sociedades sostenibles.
\end{abstract}

Palabras clave: crecimiento demográfico; emergencia planetaria; límites planetarios; población; sostenibilidad; transición demográfica; transición a la sostenibilidad

\section{The Oblivion of Demography in Sustainability's Studies}

The number of human beings has been quite small during most of the history of our species, and didn't reach a billion until the beginning of the 19th century. But since then, the world population begun to rise very quickly, surpassing 6 billion at the end of the 20th century. This extraordinary demographic explosion received logical attention from the scientific community, seriously concerned about its possible effects on the biosphere in general and, very particularly, on human societies. But nowadays, however, although the global population has surpassed 7.5 billion and continues to increase in about 80 million each year, the demographic problem has ceased to have a relevant presence in studies and publications related to the current situation of planetary emergency and the necessity of an urgent transition to sustainable societies. Our purpose in this paper is to discuss the reasons of this current lack of attention to demography that we have perceived even in journals dedicated to Sustainability Science, a new scientific domain appeared at the beginning of the 21th century with the specific aim of studying globally the ensemble of interconnected problems that may determine the unsustainable Earth's situation. We intend to analyse the reasons given to justify the lack of attention to demography in these studies on Sustainability, in order to better target the necessary transition to sustainable societies.

Keywords: demographic explosion; demographic transition; planetary emergency; planetary limits; population; sustainability; transition to sustainability.

Para citar el artículo. Gil Pérez, D. y Vilches, A. (2017). El olvido de la demografía en los estudios de Sostenibilidad. Ápice. Revista de Educación Científica, 1(2), 1-17. DOI: https://doi.org/10.17979/arec.2017.1.2.3074

Contacto.daniel.gil@uv.es, amparo.vilches@uv.es 


\section{Introducción ¿Qué se quiere decir al hablar de Sostenibilidad?}

Abordar la relación entre Demografía -o cualquier otra problemática- y Sostenibilidad exige, en primer lugar, evitar lo que Engelman (2013) denomina "sosteniblabla" (el uso abusivo y confuso del término sostenible). Se necesita para ello diferenciar los conceptos científicos de Sostenibilidad y Desarrollo Sostenible (DS) del uso trivial de estas palabras, con significados a menudo contrapuestos a dichos conceptos.

Conviene, pues, recordar que el concepto científico de Sostenibilidad -"la idea central unificadora más necesaria en este momento de la historia de la humanidad", según Bybee (1991)- surgió por vía negativa, como resultado de análisis científicos concordantes de la actual situación del mundo. Una situación que es fruto de actividades humanas generadoras de una pluralidad de graves problemas socioambientales "glocales" -a la vez locales y globales (Novo, 2006)- estrechamente vinculados y que se potencian mutuamente, por lo que ninguno de ellos puede encontrar solución aisladamente, ya sea el cambio climático, la pérdida de biodiversidad, la pobreza extrema de miles de millones de personas o cualquier otro (Vilches y Gil, 2003; Diamond, 2006). Ello ha llevado, hace ya cerca de tres décadas, a calificar esta situación, que amenaza gravemente el futuro de la humanidad, como una "emergencia planetaria" (Bybee, 1991) que exige urgentes medidas correctoras (Vilches y Gil, 2016).

La cuestión de las necesarias (y posibles) medidas correctoras es esencial para introducir y hacer comprensible el concepto de Sostenibilidad, particularmente desde la educación, evitando pesimismos paralizantes. No basta, en efecto, con hablar de problemas y de situación planetaria insostenible. Resulta también imprescindible poner énfasis en que, aunque la situación es grave y es urgente actuar, aún estamos a tiempo de adoptar las medidas necesarias para la transición a sociedades sostenibles (Sachs, 2008), entendidas -de acuerdo con la definición de Desarrollo Sostenible dada por la Comisión Mundial del Medio Ambiente y del Desarrollo (CMMAD, 1988)-como aquellas en las que se satisfacen las necesidades de la generación presente sin comprometer la capacidad de las generaciones futuras para satisfacer sus propias necesidades. La CMMAD, conviene insistir, no expresa aquí únicamente su preocupación por las generaciones futuras, sino que habla en primer lugar de satisfacer las necesidades de la generación presente; $y$, en la misma página, reclama la necesaria igualdad dentro de cada generación.

Pese a ello, lecturas muy superficiales -pero, lamentablemente, frecuentes incluso en algunos sectores del movimiento ecologista- han llevado a criticar la idea de Desarrollo Sostenible por, supuestamente, olvidar las injustas desigualdades que se dan en la actualidad entre quienes viven en un mundo de opulencia y quienes lo hacen en la mayor de las miserias. Estas críticas injustificadas llegan hasta considerar el DS "como una nueva mistificación del Norte para continuar alegremente sus prácticas de desarrollo" (Girault y Sauvé, 2008), entendido el desarrollo como crecimiento depredador. El "sosteniblablá" denunciado por Engelman (2013) se retroalimenta así con el uso distorsionado de los conceptos de Sostenibilidad y DS y con críticas superficiales a dichos conceptos. Una retroalimentación que confunde a la ciudadanía y dificulta su implicación en la transición a sociedades sostenibles.

Es frecuente, ciertamente, encontrar en el discurso empresarial, político e incluso sindical, un uso del concepto de DS como sinónimo de "crecimiento sostenido". Un crecimiento que conlleva la incorporación de recursos y la generación de residuos, 
externalizando sus inevitables consecuencias de degradación ambiental, por lo que resulta esencialmente insostenible en un planeta finito. Pero esto es algo que debe ser denunciado como una tergiversación, en muchos casos interesada (que se da también al calificar como "verdes", o "ecológicos" ciertos productos y empresas que distan mucho de serlo), en vez de ver en ello -como algunos hacen erróneamente- la esencia misma del concepto. Es preciso insistir en que el concepto de DS parte de la comprensión de que puede haber desarrollo, mejora cualitativa o despliegue de potencialidades, sin crecimiento, es decir, sin incorporación de mayor cantidad de materiales, ni producir, por tanto, más residuos (Daly, 1991). Así pues, si bien no puede haber crecimiento indefinido en un mundo finito, sí es posible un desarrollo continuado. Posible y necesario, porque las actuales formas de vida no son sostenibles y deben experimentar cambios cualitativos profundos, tanto para aquellos (la mayoría) que viven en la precariedad, como para el $20 \%$ que vive más o menos confortablemente... contribuyendo a la degradación de la biosfera. Y esos cambios cualitativos suponen un desarrollo (sin crecimiento) que es preciso (iy posible!) diseñar y orientar adecuadamente, dando respuesta a un interrogante fundamental: ¿cómo avanzar hacia sociedades sostenibles desde un mundo que sigue hoy dominado por la competitividad al servicio de intereses particulares a corto plazo (algo que ha acompañado al desarrollo de la humanidad desde sus orígenes, provocando conflictos destructivos), a lo que se añade ahora la enorme capacidad para alterar la biosfera alcanzada por nuestra especie?

Muchos otros autores, como el Premio Nobel de Física Gell-Mann (1992), han insistido en la necesidad de salir al paso de las visiones distorsionadas y empobrecidas del nuevo concepto de Sostenibilidad. Un concepto alejado del significado literal de la palabra 'sostenible'. Por ejemplo, señala Gell-Mann, la ausencia completa de vida, o de seres humanos, puede ser sostenible en el sentido habitual de la palabra, es decir, puede durar mucho tiempo, pero eso no es lo que queremos decir al hablar de Sostenibilidad.

No cabe tampoco concebir la Sostenibilidad como la persistencia de la situación actual, en la que una cuarta parte de la humanidad vive en la pobreza extrema, mientras otra cuarta parte practica un consumismo depredador; ni puede concebirse como la búsqueda de mejoras en los estándares de vida de las poblaciones hoy desfavorecidas, a costa de una creciente e insostenible degradación ambiental.

El significado de 'Sostenibilidad' y de 'Desarrollo Sostenible' podría asociarse, sostiene Gell-Mann (op. cit), al logro de un nivel de vida de calidad para el conjunto de los seres humanos, evitando las desigualdades extremas (origen de conflictos destructivos), y no poniendo en peligro a las futuras generaciones. Esta Sostenibilidad de una vida humana de calidad exige -añade Gell-Mann- calidad de la biosfera, incluyendo la supervivencia de muchos de los organismos con los que compartimos el planeta y de los sistemas ecológicos que forman.

Hemos de hacer frente, nos señalaba Gell-Mann ya en 1992 (op. cit), a una creciente multiplicidad de problemas ambientales que afectan al clima, a los océanos, a la calidad del agua y del aire, sin olvidar la deforestación, desertificación, erosión de los suelos, la desaparición de especies y, en suma, la degradación de los ecosistemas terrestres y marítimos. Muchos de estos problemas, reconocía Gell-Mann, son viejos, pero su escala es ahora muy superior, y a ello se añade un crecimiento explosivo de la población, que nunca antes se había producido y que viene a agravar muchos de los problemas señalados. 
¿Hasta qué punto está justificada esta inclusión del crecimiento demográfico entre los problemas a tener en cuenta para el logro de la Sostenibilidad? ¿Cuáles son las razones que se aducen y hasta qué punto son válidas?

\section{¿Constituye el crecimiento demográfico un problema para la Sostenibilidad?}

Como acabamos de ver, Gell-Mann (1992) consideraba la explosión demográfica como un nuevo problema que venía a potenciar el resto de problemas causantes de la situación de emergencia planetaria.

Ciertamente, nunca hasta entonces se había producido un crecimiento tan rápido de la población, debido, en buena parte, a los avances médicos y sanitarios que redujeron drásticamente los índices de mortalidad temprana. Baste señalar que hubo que esperar más de doscientos mil años -desde los orígenes de la especie sapiens hasta principios del siglo XIX- para que la población alcanzara los mil millones, mientras que a finales del siglo XX superaba ya los 6000 millones. Este crecimiento acelerado -que gráficos como el de la figura 1 (Pérez Díaz, 2010) muestran con claridad hasta el año 2000 y prolongan con estimaciones hasta 2050- no puede continuar indefinidamente, como tampoco puede continuar, añadía Gell-Mann (op. cit), el creciente agotamiento de los recursos naturales.

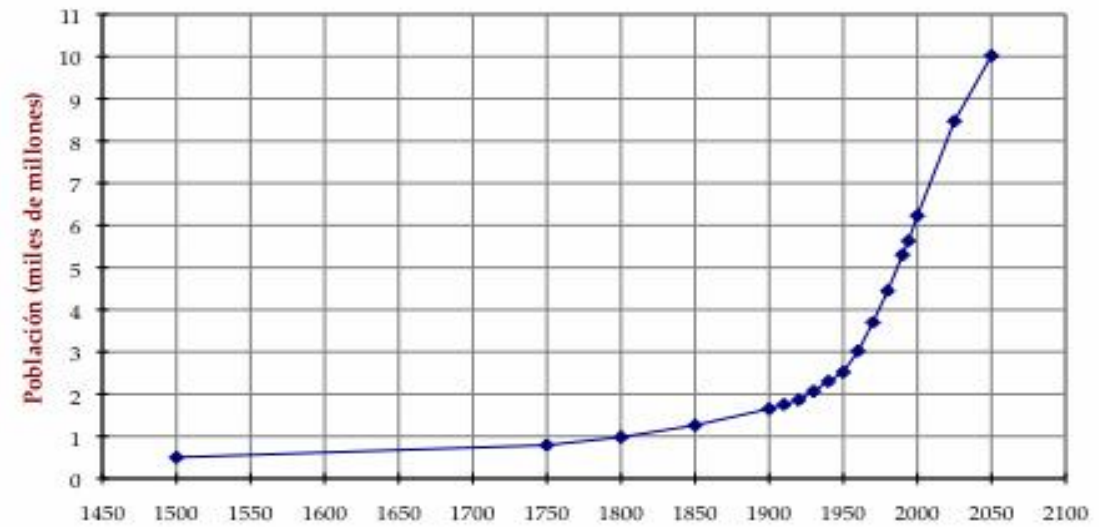

Figura 1. Estimación de la evolución de la población mundial (1500-2050)

Debe programarse pronto -insistía Gell-Mann (op. cit.)- la inflexión ordenada de estos procesos, o dicha inflexión tendrá lugar por las vías tradicionales de las guerras, las hambrunas y las epidemias. Pero, incluso si se produce el aplanamiento de la curva de crecimiento, ¿̇lo hará en un nivel que permita una calidad de vida humana razonable y la persistencia de una diversidad biológica suficientemente amplia?

La preocupación por el crecimiento demográfico era compartida a finales del siglo XX, y sigue siéndolo a principios del XXI, por numerosos expertos (CMMAD, 1988; Ehrlich y Ehrlich, 1994; Brown y Mitchell, 1998; Folch, 1998; Sartori, y Mazzoleni, 2003; Diamond, 2006; Engelman, 2012). Resumiremos aquí los argumentos dados por estos y otros autores para justificar su preocupación por el papel que el crecimiento demográfico puede estar jugando, junto al hiperconsumo de una quinta parte de la humanidad y otros problemas socioambientales, en la actual situación de emergencia planetaria:

- A lo largo del siglo XX la población se ha más que triplicado. Y aunque ahora se esté produciendo un descenso en la tasa de crecimiento de la población, esta sigue aumentando en unos 80 millones cada año, por lo que puede duplicarse de 
nuevo en pocas décadas. La Comisión Mundial del Medio Ambiente y del Desarrollo (CMMAD, op. cit.) señaló hace ya 30 años las consecuencias de este crecimiento: "En muchas partes del mundo, la población crece según tasas que los recursos ambientales disponibles no pueden sostener, tasas que están sobrepasando todas las expectativas razonables de mejora en materia de vivienda, atención médica, seguridad alimentaria o suministro de energía".

- Alrededor de un $40 \%$ de la producción fotosintética primaria de los ecosistemas terrestres es usado por la especie humana cada año para, fundamentalmente, comer, obtener madera, leña para hacer fuego, etc. Es decir, la especie humana está próxima a consumir tanto como el conjunto de las demás especies. Como escribe Jared Diamond a este respecto, "Dada la tasa de crecimiento de la población humana, y sobre todo del impacto de la población, en 1986 se preveía que a mediados de este siglo estaríamos aprovechando ya la mayor parte de la capacidad fotosintética terrestre. Es decir, la mayor parte de la energía irradiada por la luz solar se utilizará para fines humanos" (Diamond, 2006, página 636).

- Otra forma de ilustrar el "supremacismo" de la especie humana, que amenaza peligrosamente la biodiversidad, es considerar lo que supone que la masa del conjunto de más de 7500 millones de seres humanos supera ya los 300 millones de toneladas y la de los animales criados para su alimentación (gallinas, cerdos, ovejas y vacas) es de unos 700 millones de toneladas, mientras que la de la totalidad de los denominados animales salvajes (desde el puerco espín y pingüino al elefante y ballena) es inferior a 100 millones de toneladas (Hahari, 2011, capítulo 18).

- Como explicaron los expertos en Sostenibilidad, en el marco del llamado Foro de Río + 5 (Naciones Unidas, 1997), la actual población precisaría de los recursos de tres Tierras (!) para alcanzar un nivel de vida semejante al de los países desarrollados. Puede decirse, pues, que hemos superado ya la capacidad de carga del planeta, es decir, la máxima cantidad de seres humanos que el planeta puede mantener de forma permanente en condiciones de vida aceptables para todos. De hecho se ha estimado en 1,7 hectáreas la biocapacidad del planeta por cada habitante (es decir el terreno productivo disponible para satisfacer las necesidades de cada uno de los más de 7500 millones de habitantes del planeta) mientras que en la actualidad la "huella ecológica" media por habitante (área de territorio ecológicamente productivo necesaria para producir los recursos utilizados y para asimilar los residuos producidos por una población dada) es de unas 2,8 hectáreas. En definitiva, aunque se distribuyeran mejor los recursos, evitando las desigualdades inaceptables que hoy se dan, la huella ecológica del conjunto de la población superaría ampliamente la biocapacidad de la Tierra: "Incluso si consumieran, en promedio, mucho menos que hoy, los nueve mil millones de hombres y mujeres que poblarán la Tierra hacia el año 2050 la someterán, inevitablemente, a un enorme estrés" (Delibes y Delibes, 2005).

- De acuerdo con recientes estudios, alcanzar los Objetivos de Desarrollo Sostenible aprobados por la Asamblea General de Naciones Unidas en 2015 (PNUD, 2015), implicaría una población global, a finales del siglo XXI, no superior a 8.7 millardos, por debajo del límite inferior de la estimación de crecimiento demográfico prevista por Naciones Unidas (Abel, Barakat, Samir y Lutz,2016). 
- Existe una fuerte resistencia social a aceptar que el crecimiento de la población plantea un serio problema, debido al desconocimiento de los datos científicos y a prejuicios religiosos y políticos muy enraizados. Constatamos esa resistencia en el medio educativo mediante cuestionarios y entrevistas. Así, al pedir a 521 profesores de ciencias en formación que enumeraran los problemas y desafíos a los que, en su opinión, la humanidad ha de hacer frente en la actualidad y en el próximo futuro, solo un $17.7 \%$ (SD 1.7) hicieron referencia a la problemática demográfica (Gil Pérez et al., 2003). Y al repetir la experiencia en 2017 hemos obtenido resultados bastante similares: de 126 estudiantes del Máster en Profesorado de Secundaria que respondieron a la misma cuestión, solo un $22.2 \%$ (SD 3.7) se refería a dicha problemática.

Preocupaciones semejantes ante el crecimiento explosivo de la población llevaron, hace ya casi un cuarto de siglo, a Ehrlich y Ehrlich (1994), a afirmar con rotundidad que la explosión demográfica terminará muy pronto. Lo que no sabemos es si el fin se producirá de forma benévola, por medio de un descenso de las tasas de natalidad, o trágicamente, a través de un aumento de las tasas de mortalidad. Y concluían que el problema demográfico es el problema más grave al que se enfrenta la humanidad, dada la enorme diferencia de tiempo que transcurre entre el inicio de un programa adecuado y el comienzo del descenso de la población.

Diamond (op. cit.) ha cuestionado justificadamente esta tendencia a designar "el problema más grave", ya sea el crecimiento demográfico, como hacían Ehrlich y Ehrlich (op. cit.) a finales del siglo XX, o bien el cambio climático, como es frecuente escuchar $\mathrm{y}$ leer hoy en día, o cualquier otro (la acelerada pérdida de biodiversidad, la pobreza extrema de millardos de seres humanos, etc.). Diamond analiza las circunstancias que se dieron en el colapso relativamente brusco de algunas sociedades aisladas -como la cultura amerindia de los Anasazi- y muestra que dicho colapso ha estado asociado a una docena de problemas, entre los cuales aparece, ciertamente, la explosión demográfica que se produjo localmente, aunque junto a otros problemas como el agotamiento de recursos, la acumulación de residuos o la degradación de los hábitats naturales. Pero quizás lo más interesante de su estudio sea la comprensión de que todos esos problemas aparecen estrechamente vinculados, de forma que "si no resolvemos cualquiera de la docena de problemas sufriremos graves perjuicios (...) porque todos ellos se influyen mutuamente" (Diamond, op. cit., página 645). Dicho con otras palabras: ninguno de los problemas es "más importante" que los otros, puesto que ninguno puede resolverse aisladamente, sino que es preciso actuar sobre el conjunto de problemas interconectados. Su conclusión, sin embargo, es muy similar a la de Ehrlich y Ehrlich (op. cit.), con solo sustituir "explosión demográfica" por "problemas medioambientales", pues en la misma página Diamond escribe: "los problemas medioambientales del mundo se resolverán, de un modo $u$ otro, en el curso de la vida de los niños y jóvenes de hoy día. La única pregunta es si acabarán resolviéndose de una forma agradable y escogida por nosotros mismos o de formas desagradables que no hayan sido fruto de nuestra elección, como las guerras, el genocidio, las hambrunas, las enfermedades epidémicas o la desaparición de sociedades. Aunque todos estos fenómenos nefastos han sido endémicos para la humanidad a lo largo de toda nuestra historia, su frecuencia aumenta con la degradación ambiental, la presión demográfica y la consiguiente pobreza e inestabilidad política".

Se puede y se debe discrepar, pues, de que el crecimiento demográfico constituya "el problema más grave", pero cabe reconocer, como indican Delibes y Delibes (2005), que 
se superponen los dos factores que están asociados de forma permanente e indisoluble al impacto de la humanidad sobre el ambiente: de un lado, el derroche de los más ricos, y de otro, el enorme tamaño de la población mundial. Se trata de "bombas de relojería con mechas de menos de 50 años" (Diamond, op. cit., página 643).

El reto definitorio del siglo XXI, señala Sachs (2008, p. 17), será afrontar la realidad de que la humanidad comparte un destino común en un planeta superpoblado: "El siglo XXI echará por tierra muchas de nuestras convicciones fundamentales acerca de la vida económica (...) Los desafíos del desarrollo sostenible (preservar el medio ambiente, estabilizar la población mundial, reducir la brecha entre ricos y pobres y poner fin a la pobreza extrema) ocuparán el centro de la escena. La cooperación global deberá pasar a un primer plano. La idea misma de que los estados-nación compitan por los mercados, la energía y los recursos quedará anticuada".

Brown y Mitchell (1998) resumían la cuestión a finales del siglo XX insistiendo en que la estabilización de la población es un paso fundamental para detener la destrucción de los recursos naturales y garantizar la satisfacción de las necesidades básicas de todas las personas. En el mismo sentido se había ya pronunciado, una década antes la Comisión Mundial del Medio Ambiente y del Desarrollo (CMMAD, op. cit.), afirmando que la reducción de las actuales tasas de crecimiento demográfico es absolutamente necesaria para lograr un desarrollo sostenible. Una condición necesaria, pero no suficiente: los mismos Ehrlich y Ehrlich (op. cit.) que, como ya hemos señalado, se referían al crecimiento demográfico como "el principal problema ecológico", también llamaron la atención sobre el hecho de que la superpoblación de los países ricos, desde el punto de vista de la habitabilidad de la Tierra, es una amenaza más seria que el rápido crecimiento demográfico de los países pobres. Es el mundo rico, ya superpoblado, el que tiene un consumo per cápita muy superior al de los africanos y el que más contribuye, por tanto, al agotamiento de los recursos, a la lluvia ácida, al calentamiento del globo, a la crisis de los residuos, etc.

Se puede seguir fundamentando la incidencia del crecimiento demográfico, junto a otros problemas, en la actual situación de emergencia planetaria, con la ayuda de numerosas referencias que vienen a reforzar los argumentos ya presentados (Bryant, Carver, Butler y Anaged, 2009; Moran, 2010; Bradshaw y Brook, 2014; Ceballos, Ehrlich y Dirzo, 2017), pero es igualmente importante analizar además los argumentos de quienes cuestionan esa incidencia e incluso llegan a afirmar que el problema es justo el contrario: el descenso de la tasa de crecimiento de la población mundial. Lo haremos en el siguiente apartado.

\section{Cambios en la percepción de la problemática demográfica: la transición demográfica y la preocupación por el descenso de los índices de natalidad}

Las llamadas de atención que acabamos de presentar acerca de los peligros generados por la explosión demográfica contrastan con la actual falta de atención generalizada a dicha problemática (Engelman, 2009). Una falta de atención que afecta incluso a la consideración de las 169 Metas y 17 Objetivos de Desarrollo Sostenible (PNUD, op. cit.) en los que no aparece explícitamente ninguna referencia al problema de la población ni a las medidas que se deberían adoptar. En particular, esta falta de atención es patente en los órganos de expresión de la nueva Ciencia de la Sostenibilidad, surgida a principios del siglo XXI con el propósito expreso de realizar el tratamiento sistémico, sin reduccionismos ni olvidos, del conjunto de problemas que determinan la insostenible situación actual del planeta (Kates et al., 2001; Vilches y Gil Pérez, 2013). Baste señalar, 
por ejemplo, que el análisis -que hemos realizado como parte de este trabajo- del contenido de más de cien artículos de la revista Sustainability Science, desde su aparición en 2006, nos lleva a concluir que la atención a la problemática demográfica ha sido nula en su primera década de existencia.

Igualmente significativa es la ausencia de referencias a la demografía al plantear la cuestión de los límites planetarios que, como explica Folke (2013, página 55), "En conjunto describen un marco donde la humanidad puede desenvolverse con seguridad y que, de respetarse, garantizaría probablemente que la Tierra pueda permanecer en un estado similar al Holoceno". Nueve son los límites planetarios señalados: cambio climático, diversidad biológica, fijación de nitrógeno y de fósforo, reducción del ozono estratosférico, acidificación de los océanos, utilización de agua dulce, cambios en los usos del suelo, contaminación atmosférica por aerosoles y contaminación química. Y aunque se reconoce que "Los límites planetarios son interdependientes, pues vulnerar uno de ellos puede hacer que varíen otros límites o que estos sean superados a su vez" (Folke, op. cit., página 60), se ignora su lógica vinculación con el tamaño de la población humana y los niveles de consumo.

Esta reciente falta de atención a la demografía en los estudios sobre Sostenibilidad podría ser debida al convencimiento de que hemos entrado en una fase de "transición demográfica" que pone fin al peligro de un crecimiento explosivo de la población. Merece la pena, pues, detenerse en analizar esta transición.

Ejemplificaremos lo que se entiende por transición demográfica refiriéndonos a lo sucedido desde fines del siglo XVIII en Europa: en esa época, cada mujer tenía seis hijos por término medio, pero la población apenas aumentaba, porque buen número de esos niños y niñas no llegaba a la edad adulta y porque los adultos se veían afectados también por numerosas enfermedades causantes de muerte temprana. Pero con la mejora de las condiciones de vida que acompañó a la revolución industrial $-y$, no debemos olvidar, a la explotación de las colonias-, tales como una mejor nutrición y saneamiento, así como a progresos médicos como la invención de las vacunas, etc., la mortalidad infantil decreció y la esperanza de vida aumentó; y como las parejas siguieron teniendo muchos hijos, se produjo un crecimiento explosivo de la población. Algo similar -reforzado por los procesos migratorios- tuvo lugar en lo que se denominó "neoeuropas" o "vástagos de Occidente" como Australia, Canadá o Argentina y, muy particularmente, Estados Unidos, cuya población se multiplicó por diez en el siglo XIX (Osterhammel, 2015, capítulo 4). Al cabo de varias generaciones, sin embargo, perdido el temor a la frecuente muerte de los hijos, que había dejado de producirse, las parejas pasaron a tener menos hijos, lo que se vio también favorecido por la incorporación de la mujer a la actividad laboral fuera del hogar y el acceso a medios para evitar embarazos no deseados. De este modo, las tasas de nacimientos y muertes volvieron a aproximarse poniendo fin a la explosión demográfica en una transición de duración variable en los distintos países: 200 años en Inglaterra (1740-1940), 70 en Alemania (1870-1940) y 40 en Japón (1920-1960) (Osterhammel, 2015, capítulo 4). Pero durante ese periodo de transición demográfica, la Europa Industrial se había superpoblado y envejecido. Algo similar está ocurriendo hoy en otros países, por lo que cabe esperar, ciertamente, que se interrumpa el crecimiento de la población mundial... aunque no sin antes producirse -como ocurrió en Europa y en las "neoeuropas"- la sobrepoblación de regiones menos pobladas como el África subsahariana.

Robinson (2013) ha analizado estos cambios demográficos. Comienza recordando que durante las décadas precedentes una preocupación dominante había sido que el rápido 
crecimiento de la población iba a agravar la pobreza, provocar inestabilidad política y agotar los recursos naturales. Cabría pensar, pues, que el hecho de que la población mundial crezca ahora más lentamente -debido a la transición demográfica caracterizada por un rápido descenso voluntario de la tasa de fertilidad en un número creciente de países- iba a ser saludado positivamente. No ha sido así, aclara Robinson (op. cit.): la transición demográfica -que algunos denominan "implosión poblacional" (pese a que la población mundial sigue creciendo)- es presentada por los medios de comunicación y muchos comentadores (en general no demógrafos) como algo tan amenazador, para nuestro futuro económico y social, como lo era la "explosión demográfica" algunas décadas antes.

Se trata, en efecto, de una preocupación frecuentemente aireada por los medios de comunicación, que expresan el temor de que, debido a la baja tasa de natalidad, la proporción entre el número de personas en edad laboral y quienes tienen 65 o más años, descienda muy rápidamente, haciendo insostenible el sistema de pensiones. En consecuencia se reclaman políticas de impulso de la natalidad para lograr que esta proporción se mantenga en valores compatibles con las ayudas a quienes no trabajan ( 4 o 5 trabajadores por pensionista). Así, por ejemplo, el diario "El País", en su número del 7 de octubre de 2010, publicó "Las claves del sistema de pensiones", haciéndose eco del peligro que el descenso de la natalidad conlleva para la viabilidad del sistema de pensiones: "el descenso en la natalidad y el incremento de esperanza de vida provocará que la población mayor de 65 años frente a la que está en edad de trabajar se dispare en los próximos años y décadas hasta llegar al 60\% de aquí a 2060. Según Bruselas, si ahora hay cuatro cotizantes por cada jubilado con pensión contributiva, dentro de 50 años esta proporción se reducirá a la mitad". Y en el mismo sentido, el periódico "La Vanguardia" publicaba el 30 de mayo de 2014 el editorial "Alarmante descenso de la natalidad", en el que se afirma "Los problemas que crea esta situación son obvios, y quizás el principal sea poner en peligro las pensiones de los jubilados".

Debemos hacer notar, sin embargo, que pensar en el mantenimiento de una proporción de 4 o 5 trabajadores por pensionista, como se hace en estos editoriales, es un ejemplo de planteamiento centrado en el "aquí y ahora" que no considera las consecuencias a medio plazo, pues cabe esperar que la mayoría de esos "4 o 5 trabajadores" aspiren también a ser pensionistas, lo que exigiría volver a multiplicar el número de trabajadores, etc. Ello no es sostenible ni siquiera recurriendo a la inmigración, pues también esos inmigrantes habrán de tener derecho a ser pensionistas. Tales planteamientos son un auténtico ejemplo de las famosas estafas "en pirámide" (nunca mejor dicho cuando se trata de la pirámide poblacional), condenadas a producir una bancarrota global, y constituyen una buena muestra de cómo los análisis parciales, manejando datos puntuales, conducen a conclusiones erróneas. Como señala Sachs (2008, pp. 219-220), "Aunque la tasa de crecimiento demográfico del mundo ha descendido, toda complacencia en relación con este tema está fuera de lugar. La población mundial continúa incrementándose a marchas forzadas y en las regiones con menor capacidad para garantizar la salud, la estabilidad y la prosperidad de la población (...) El mundo debería adoptar un conjunto de medidas que contribuyeran a estabilizar la población mundial, mediante decisiones voluntarias, en una cifra en torno a los ocho mil millones de habitantes, en lugar de mantener la actual trayectoria que probablemente nos situará en los nueve mil millones o más en el año 2050".

Podemos hablar así de la necesidad de una "Nueva cultura demográfica", tan necesaria para la transición a la Sostenibilidad como la "Nueva cultura energética", la "Nueva 
cultura del agua", etc.; una cultura demográfica que tenga en cuenta la estrecha vinculación de los problemas y su carácter glocal (a la vez global y local), evitando los planteamientos localistas y a corto plazo, lo que obliga a transformar la actual pirámide poblacional, con muchos más jóvenes que personas ancianas -insostenible porque exige el crecimiento permanente de la población- en aproximadamente un cilindro de crecimiento cero, con números similares de seres humanos en los distintos grupos de edad. Como explica Engelman (2012, páginas 241-242), hay que "adaptarse al envejecimiento de la población en vez de intentar retrasarlo a través de incentivos o programas gubernamentales destinados a aumentar la natalidad (...) Incluso si los actuales responsables de las políticas pudieran incrementar el aumento de la población fomentando índices de natalidad más elevados o la inmigración, tendrían que lidiar con los problemas del envejecimiento en un futuro".

¿Qué sentido tiene, por otra parte, esta preocupación por la disminución de la población en edad laboral, cuando estamos asistiendo, en numerosos países, al crecimiento generalizado de la población desempleada y del trabajo precario, sobre todo entre los jóvenes? El problema hoy -y en el futuro que dibuja la innovación tecnológica y la informatización (Frey y Osborne, 2017)- no es la falta de mano de obra, sino la de puestos de trabajo (Vilches y Gil Pérez, 2016). La Organización Internacional del Trabajo (OIT), agencia de Naciones Unidas en la que están ampliamente representados los sindicatos, dirige actualmente buena parte de sus esfuerzos a luchar contra el creciente desempleo, reivindicando políticas de "trabajo decente" (Poschen, 2017) como parte de la Agenda 2030 para la promoción de los Objetivos de Desarrollo Sostenible, aprobados en 2015, como ya hemos señalado, por la Asamblea General de Naciones Unidas.

Abordaremos esta cuestión fundamental en las consideraciones finales. Antes, sin embargo, debemos referirnos a otro serio obstáculo para un adecuado planteamiento de la relación demografía-Sostenibilidad: las barreras existentes en numerosos países al acceso a la educación y a los servicios de salud sexual y reproductiva necesarios para poder ejercer el derecho a una maternidad y paternidad responsables.

\section{El derecho a una maternidad y paternidad responsables}

Hoy, cuando ya hemos sobrepasado los siete mil quinientos millones de habitantes en el planeta, es necesario centrar la atención en uno de los requisitos fundamentales para poder abordar adecuadamente el problema demográfico: el reconocimiento del derecho humano básico de determinar libre y responsablemente el número y el espaciamiento de los hijos, accediendo a la información y procedimientos de planificación familiar compartida entre mujeres y hombres, así como a los servicios de salud sexual y reproductiva necesarios para poder ejercer este derecho (Engelman, 2012).

El 11 de julio de 2012, en el Día Mundial de la Población, cuyo lema era ese año "Acceso universal a los servicios de salud reproductiva", Ban Ki-moon, como Secretario General de Naciones Unidas, declaró: “En este Día Mundial de la Población, hago un llamamiento para que los Estados Miembros adopten con urgencia medidas concertadas para reducir la disparidad que existe entre la demanda y la oferta de servicios de salud reproductiva". Ban Ki-moon tomaba en consideración informaciones proporcionadas por el Fondo de Población de las Naciones Unidas (UNFPA), como que más de 200 millones de mujeres, deseando evitar o posponer el embarazo, no tienen acceso a una planificación familiar efectiva; que centenares de mujeres mueren cada día durante el parto; que millones de jóvenes que inician su etapa reproductiva carecen a menudo de la información, los 
conocimientos y los servicios que necesitan para protegerse a sí mismos. Por ello Ban Kimoon insistía en que "La salud y los derechos reproductivos son esenciales para el desarrollo sostenible y la reducción de la pobreza. La inversión en el acceso universal a la salud reproductiva es una inversión crucial para lograr sociedades saludables y un futuro más sostenible". En 2017, el lema ha sido "Planificación familiar, para empoderar a las personas y contribuir al desarrollo de los países" insistiendo en la importancia del acceso voluntario a métodos seguros de planificación familiar, un derecho humano clave para alcanzar la igualdad de género y el empoderamiento de las mujeres.

Es preciso, en particular, acabar con los 16 millones de embarazos de menores de edad que dan a luz cada año y con los más de 3 millones de abortos clandestinos de adolescentes que se realizan sin las debidas garantías, poniendo en riesgo sus vidas. Por ello, el Día Mundial de la Población de 2013 estuvo dedicado a "El embarazo en la adolescencia" y Ban Ki-moon señaló: "Si dedicamos atención y recursos a la educación, la salud y el bienestar de las adolescentes, estas se transformarán en una fuerza aún más poderosa para el cambio positivo de la sociedad, que tendrá efectos en las generaciones venideras. En este Día Mundial de la Población, comprometámonos a apoyar a las adolescentes para que puedan hacer realidad su potencial y contribuir a nuestro futuro común". Y ese mismo año, el Fondo de Población de Naciones Unidas, proponía: "Debemos asegurarnos de que todos los embarazados son deseados, todos los nacimientos son seguros y que todos los niños pueden desarrollar su potencial". Y lanzaba el eslogan "By Choice, not by Chance", es decir, "Por elección, no por azar". Por elección responsable, atendiendo al bienestar de los nuevos seres y del conjunto de la especie humana. No por azar, ni por imposición de fundamentalismos religiosos que persisten en vincular sexualidad a procreación.

Particularmente grave es la influencia que muchas tradiciones religiosas han tenido y siguen teniendo en el enfoque de la problemática demográfica. Si el "Creced y multiplicaos" bíblico tenía sentido en periodos históricos en los que la población humana era muy baja y se corría el riesgo de la desaparición de la especie, no es sensato hoy ignorar la gravedad de la rápida multiplicación de la población humana y la imposibilidad de que este crecimiento demográfico continúe indefinidamente. Baste recordar que, como ya hemos señalado, la población humana actual está ya próxima a consumir cada año tanta producción fotosintética primaria como el conjunto de las demás especies, lo que supone un indudable ataque al equilibrio de la biosfera y está contribuyendo a provocar extinciones masivas que, de continuar, arrastrarían consigo a la propia especie humana.

Tampoco tiene sentido la condena absoluta de las interrupciones voluntarias del embarazo o de la experimentación con embriones, presente incluso en un documento tan reciente como la encíclica papal "Laudato Si", hecha pública en 2015. Esta práctica de la experimentación con embriones humanos y la despenalización del aborto son atribuidas en la encíclica (ítem 136) al hecho de que "cuando la técnica desconoce los grandes principios éticos, termina considerando legítima cualquier práctica", pero es preciso señalar que se basa en estudios científicos con una profunda fundamentación ética: parte de la consideración de que las células que forman el embrión -inicialmente las mismas que existían por separado en los espermatozoides y óvulos- no pasan abruptamente de ser simple materia orgánica, a ser personas con derechos y a merecer protección desde el momento mismo de la fecundación, ya que no tienen todavía capacidad de sentir, sufrir y gozar (y tardarán meses en empezar a tenerla). Quienes sí sufren son las mujeres embarazadas contra su voluntad. Y los principios éticos exigen 
ayudarles, ayudar a erradicar las barreras educativas y legislativas que se oponen a una vida afectiva y sexual satisfactoria y a una maternidad y paternidad responsables, contribuyendo así a la superación del conjunto de graves problemas socioambientales a los que se enfrenta la humanidad. Por eso es imprescindible reclamar en todos los ámbitos la desaparición de las leyes que criminalizan los medios mal llamados "anticonceptivos". Medios gracias a los cuales las concepciones pueden ser el fruto de decisiones responsables y no la consecuencia indeseada del desconocimiento o de la imposición de fundamentalismos que exigen asociar sexualidad exclusivamente a procreación.

\section{Consideraciones finales para un adecuado planteamiento de la relación demografía-Sostenibilidad}

Podríamos concluir, a la luz de lo discutido hasta aquí, que el problema demográfico incide, ciertamente, en la actual insostenibilidad de las sociedades humanas y debe, pues, ser incorporado en el estudio global de los factores que afectan a la misma, corrigiendo la insuficiente atención que esta relación está recibiendo recientemente (Engelman, 2009).

Por lo que respecta a las propuestas fundamentadas de tratamiento, apuntan a lograr que la transición demográfica se produzca y termine lo antes posible, es decir, a conseguir que el número de nacimientos por pareja deje de sobrepasar la tasa de reposición, evitando al propio tiempo que la población se estabilice en cifras superiores a la capacidad de carga del planeta, algo que nuestra civilización, probablemente, no podría resistir. Ello implica que durante un cierto tiempo la pirámide poblacional se invierta y crezca más el número de ancianos que el de jóvenes. Es más, puede llegar a ocurrir que el número de muertes llegue a superar provisionalmente al de nacimientos y que la población solo se estabilice tras una cierta disminución.

Todo ello plantea, sin duda, serios problemas que deben ser previstos. De hecho, países que se enfrentan al descenso de la natalidad y al envejecimiento de la población, como Japón, Corea del Sur o Alemania, han empezado a invertir en robótica avanzada y construcción de androides, para paliar la prevista falta de mano de obra (Chamie, 2017). En cualquier caso, los problemas derivados del envejecimiento poblacional son menores que los que conlleva el crecimiento indefinido de la población -algo absolutamente insostenible- o su estabilización en cifras demasiado elevadas para garantizar la sostenibilidad de los recursos y ecosistemas, así como el necesario mantenimiento de la biodiversidad, poniendo fin a las actuales extinciones masivas que, de continuar, arrastrarían consigo a la propia especie humana. En definitiva, el bienestar durable del conjunto de los seres humanos exige que la actual transición demográfica estabilice la población en cifras no muy superiores a las actuales.

Debemos recordar, sin embargo, que este requisito, si bien imprescindible, es totalmente insuficiente... porque no existe un problema demográfico que podamos abordar y resolver aisladamente, sino que forma parte de la problemática mucho más amplia y compleja de la actual insostenibilidad de nuestras formas de vida y de la urgente necesidad de transición a sociedades sostenibles. Una transición que exige numerosas y simultáneas transiciones, además de la transición demográfica que aquí estamos estudiando. Entre otras, son necesarias las siguientes:

- Transición a un consumo responsable, poniendo fin al actual ritmo de utilización de todo tipo de recursos esenciales (agua dulce, cubierta vegetal, bancos de 
pesca, etc.), muy superior al de su regeneración, cuando son renovables, o al de su sustitución por otros que sí lo sean. Como escribe Yuval Noah Harari en su "Brief History of Humankind", A lot of evidence indicates that we are destroying the foundations of human prosperity in an orgy of reckless consumptions (Harari, 2011, p. 424).

- Transición a una economía circular, que evite la producción continuada de residuos contaminantes, muy superior en la actualidad a la capacidad del planeta para digerirlos, por lo que una contaminación pluriforme y sin fronteras envenena suelos, ríos, mares y aire y afecta ya a todos los ecosistemas.

- Transición energética, para pasar del uso de los recursos fósiles a las energías renovables, evitando el acelerado incremento de la concentración de gases de efecto invernadero en la atmósfera y océanos, que está provocando un desarreglo climático visible ya, entre otras muchas consecuencias, en la rápida disminución de las llamadas nieves perpetuas (la más importante reserva de agua dulce con la que cuentan miles de millones de seres humanos) y en el aumento de la frecuencia e intensidad de fenómenos atmosféricos extremos (huracanes, inundaciones, sequías e incendios...) que contribuyen a un proceso de degradación generalizada que corre el riesgo de llegar a ser irreversible, haciendo inhabitable la Tierra para la especie humana.

- Transición urbana, que ponga fin al proceso de urbanización acelerada y desordenada, que se acompaña del abandono del mundo rural y que potencia los efectos de la contaminación (a causa del transporte, calefacción, acumulación de residuos, etc.) y del agotamiento de recursos (por la destrucción de terrenos agrícolas, el aumento de los tiempos de desplazamiento y consiguiente consumo de recursos energéticos, etc.).

- Transición del antropocentrismo al biocentrismo, para acabar con la acelerada pérdida de biodiversidad, que obliga a hablar de una sexta gran extinción ya en marcha, que amenaza con romper los equilibrios de la biosfera y arrastrar a la propia especie humana, causante de esta extinción.

- Transición de la imposición de la uniformidad a la protección e impulso de la diversidad cultural. La actual pérdida de diversidad cultural y, muy en particular, de culturas campesinas milenarias, es tan grave como la pérdida de biodiversidad. No debe olvidarse que la diversidad de culturas es la garantía de una pluralidad de respuestas a los problemas a los que ha de hacer frente la humanidad. Cada cultura no es solo una riqueza para el pueblo que la ha creado, sino un patrimonio de toda la humanidad.

- Transición laboral. Una sociedad sostenible, sin desequilibrios insolidarios, tiene como uno de sus requisitos fundamentales la existencia de puestos de trabajo digno para el conjunto de la ciudadanía (Poschen, 2017). En un momento en que la innovación tecnológica favorece la supresión de numerosas tareas simples y de los correspondientes empleos (Frey y Osborne, 2017), se necesitan y son posibles nuevos yacimientos de ocupación que contribuyan a la transición a sociedades sostenibles y a satisfacer los derechos fundamentales de ciudadanas y ciudadanos (Vilches y Gil-Pérez, 2016; Gil Pérez y Vilches, 2017). Cabe pensar así en el desarrollo de las energías renovables, la edificación ecológica, la protección y restauración de los ecosistemas, etc., sin olvidar la ocupación destinada a garantizar el acceso a servicios públicos fundamentales (salud, educación...) y a 
potenciar la producción y difusión cultural, como alternativa al insostenible consumismo de bienes materiales (Maaluf, 2009).

- Transición de la competitividad a la cooperación y universalización de los Derechos Humanos. Es insostenible un sistema socioeconómico guiado por la búsqueda del máximo beneficio particular a corto plazo, que apuesta por un crecimiento económico y demográfico indefinido, insostenible en un planeta finito, sin atender a sus consecuencias ambientales y sociales, lo que le convierte en responsable de los problemas que hemos ido enumerando aquí y de otros igualmente graves y estrechamente vinculados, como hambre, dolorosas migraciones y conflictos y violencias causados por la competitividad, por el afán de controlar los recursos energéticos y otras materias primas esenciales $y$, en suma, por la destructiva anteposición de intereses particulares a la cooperación en beneficio de todos y de las generaciones futuras.

Esta transición a la Sostenibilidad no debe verse como un sacrificio, como una obligada pérdida de nivel de vida para todos aquellos que hoy disfrutan de un cierto consumo material. Muy al contrario, como explica Maaluf (2009, páginas 205-206), "No se trata de imponerse privaciones ni practicar la ascesis. (...) Pero si deseamos disfrutar durante mucho tiempo y con plenitud de cuanto nos brinda la vida no nos queda más remedio que modificar nuestra forma de comportarnos. No para mermar nuestra paleta de sensaciones, sino, antes bien, para ampliarla, para enriquecerla, para buscar otras satisfacciones que podrían resultar intensas (...) ¿ ¿No distinguimos, acaso, en lo referente a las fuentes de energía, entre la energía fósil, que se agota y contamina, y las energías renovables, como las olas, la energía eólica o la geotérmica, que no se agotan? Podrían hacerse distinciones así al hablar de nuestro modo de vida. Podemos intentar satisfacer las necesidades y gozar de los placeres de la existencia consumiendo más, lo que supondrá una carga para los recursos del planeta y acarreará tensiones destructivas. Pero también podríamos hacerlo de otra manera, haciendo prevalecer el aprendizaje en todas las edades de la vida, animando a todos nuestros contemporáneos a que estudien lenguas, a que se apasionen por las disciplinas artísticas, a que se familiaricen con las diversas ciencias, para que así sean capaces de valorar lo que significa un descubrimiento en biología o en astrofísica. El saber es un universo inconmensurable; todos podríamos pasarnos la vida tomando cosas de él sin tasa y no lo agotaríamos". Por nuestra parte añadimos que no se trata solo de proponer el consumo de conocimientos y productos culturales, en vez del consumo material: contribuir a ampliar el conocimiento en cualquiera de sus formas, elaborar productos culturales, contribuir a concebir y resolver problemas, junto a otros, puede dar satisfacciones aún mayores que el simple consumo.

Estas son cuestiones esenciales de Transición y Educación para la Sostenibilidad que se deben tener en cuenta en la formación del profesorado $y$, en general, en la alfabetización científica de la ciudadanía para su participación en la necesaria y urgente transición a sociedades sostenibles, tal como plantea la Agenda de Desarrollo 2030 para avanzar en la consecución de los Objetivos de Desarrollo Sostenible (ODS), a los que ya hemos hecho reiterada referencia: "Los 17 Objetivos de Desarrollo Sostenible y las 169 metas que anunciamos hoy demuestran la magnitud de esta ambiciosa nueva Agenda universal". La consecución de dichos ODS interconectados requiere la colaboración de todos, instituciones, gobiernos, sociedad civil... y el compromiso de una educación de calidad, igualitaria e inclusiva, a la que se dedica específicamente el Objetivo 4 y que impregna el conjunto de la Agenda. Es preciso, por tanto, que toda la educación, tanto 
formal como no reglada, contribuya a proporcionar a la ciudadanía una percepción correcta de los problemas socioambientales actuales y a fomentar actitudes y comportamientos favorables para una urgente transición a la Sostenibilidad.

Terminaremos reconociendo que esta transición a la Sostenibilidad no es algo sencillo de lograr, puesto que exige un profundo cambio del modelo socioeconómico, de nuestros hábitos $y$, en definitiva, de la forma de relacionarnos los seres humanos con la naturaleza y entre nosotros mismos. No es sencillo, pero sí es posible... y es absolutamente necesario y urgente para garantizar la continuidad de nuestra especie y unas condiciones dignas de vida para el conjunto de los seres humanos.

\section{Referencias bibliográficas}

Abel, G.J., Barakat, B., Samir, K.C y Lutz, W. (2016). Meeting the Sustainable Development Goals leads to lower world population growth. PNAS, 2016.201611386. Recuperado de: www.pnas.org/cgi/doi/10.1073/pnas. 1611386113

Bradshaw, C.J.A. y Brook, B.W. (2014). Human population reduction is not a quick fix for environmental problems. PNAS, vol. 111, No. 46, 16610-16615.

Brown, L. R. y Mitchell, J. (1998). La construcción de una nueva economía. En Brown, L. R., Flavin, C. y French, H. La situación del mundo 1998. Barcelona: Ed. Icaria.

Bryant, L., Carver, L., Butler, C. D. yAnaged, A. (2009). Climate change and family planning: least-developed countries define the agenda, Bulletin World Health Organization, 87, 852-857.

Bybee, R. W. (1991). Planet Earth in crisis: how should science educators respond? The American Biology Teacher, 53 (3), 146-153.

Ceballos, G., Ehrlich, P.R. y Dirzo, G. (2017). Biological annihilation via the ongoing sixth mass extinction signaled by vertebrate population losses and declines. PNAS I E6089-E6096. Recuperado de: www.pnas.ord/cgi/doi/10.1073/pnas.1704949114

Chamie, J. (2017). Robots: A Solution to Declining and Aging Populations? Inter PressService, September 5. Recuperado de: http://www.ipsnews.net/2017/09/ robots-solution-declining-aging-populations/

Comisión Mundial del Medio Ambiente y del Desarrollo (1988). Nuestro Futuro Común. Madrid: Alianza.

Daly, H. (1991). Steady-State Economics. Washington D.C.: Island Press.

Delibes, M. y Delibes de Castro, M. (2005). La Tierra herida. ¿Qué mundo heredarán nuestros hijos? Barcelona: Destino.

Diamond, J. (2006). Colapso. Barcelona: Debate.

Ehrlich, P.R. y Ehrlich, A.H. (1994). La explosión demográfica. El principal problema ecológico. Barcelona: Salvat.

Engelman, R. (2009). Population and Sustainability: Can We Avoid Limiting the Number of People? Scientific American, June 1. Recuperado de: https://www.scientificamerican.com/article/population-and-sustainability/

Engelman, R. (2012). Nueve estrategias para no alcanzar una población de 9000 millones. En Worldwatch InstituteLa situación del mundo 2012. Hacia una 
prosperidad sostenible. Barcelona: Icaria. (Capítulo 9).

Engelman, R. (2013). Más allá de la sosteniblablá. En Worldwatch Institute La situación del mundo 2013. ¿Es aún posible lograr la sostenibilidad? Barcelona: Icaria.

Folch, R. (1998). Ambiente, emoción y ética. Barcelona: Ed. Ariel.

Folke, C. (2013). Respetar los límites del planeta y recuperar la conexión con la biosfera. En Worldwatch Institute, The State of the World 2013: Is Sustainability Still Possible?New York: W.W. Norton. Capítulo 2.

Frey, C. B. y Osborne, M. A. (2017).The future of employment: How susceptible are jobs to computerisation? Technological Forecasting and Social Change, 114, 254-280.

Gell-Man, M. (1992).Visions of a Sustainable World, Engineering \&Science, Spring 1992, 5-10.

Gil Pérez, D. y Vilches, A. (2017). Educación para la Sostenibilidad y educación en derechos humanos: dos campos que deben vincularse. Teoría de La Educación. Revista Interuniversitaria, 29 (1), 79-100.

Girault, Y. y Sauvé, L. (2008). L'éducation scientifique, l'éducation à l'environnement et l'éducation pour le développement durable. Aster, 46, 7-30.

Harari, Y. N. (2011). Sapiens. A Brief History of Humankind. London: Vintage. Penguin Random House. [Existe traducción al castellano, publicada por la editorial Debate en 2015].

Kates, R. W., Clark, W.C.,Corell, R., Hall, J. M., Jaeger, C.C., Lowe, I.,Mccarthy, J. J., Schellnhuber, H. J.,Bolin, B., Dickson, N. M., Faucheux, S.,Gallopin, G. C.,Grübler, A.,Huntley, B.,Jagër, J.,Jodha, N. S., Kasperson, R. E., Mabogunje, A., Matson, P., Mooney, H., Moore, B. lii.,O'riordan, T. \&Svedin, U. (2001). Sustainability Science, Science 27 April 2001, Vol. 292 no. 5517, 641-642.

Maaluf, A. (2009). El desajuste del mundo. Cuando nuestras civilizaciones de agotan. Madrid: Alianza Editorial.

Moran, E. F. (2010). Environmental Social Science: Human-Environment Interactions and Sustainability. West Sussex, UK: Wiley-Blackwell.

Naciones Unidas (1997).La cumbre para la tierra + 5 en 1997. Recuperado de: http://www.un.org/spanish/conferences/cumbre\&5.htm)

Novo, M. (2006). El desarrollo sostenible. Su dimensión ambiental y educativa. Madrid: UNESCO-Pearson. Capítulo 3.

Osterhammel, J. (2015). La transformación del Mundo. Barcelona: Planeta.

Pérez Díaz, J. (2010) Apuntes de Demografía. CSIC. Ejercicio 3a. Población mundial (evolución). Recuperado de: https://apuntesdedemografia.com/curso-dedemografia/ejercicios-resueltos/ejercicio-3-poblacion-mundial-grafico-de-suevolucion/

PNUD (2015). Objetivos de Desarrollo Sostenible. Recuperado de: http://www.undp.org/ content/undp/es/home/sustainable-development-goals/

Poschen, P. (2017).Trabajo decente, empleos verdes y economía sostenible: Soluciones para el cambio climático y el desarrollo sostenible. Madrid: Plaza y Valdés Editores y OIT. [Publicado inicialmente en inglés, en 2015, con el título Decent Work, Green 
Jobs and the Sustainable Economy]

Robinson, W. C. (2013). Population and Sustainability. Resources for the Future (RFF), 182, Winter 2013.

Sachs, J. (2008). Economía para un planeta abarrotado. Barcelona: Debate.

Sartori, G. y Mazzoleni, G. (2003). La Tierra explota. Superpoblación y Desarrollo. Madrid: Taurus.

Vilches, A. y Gil, D. (2003). Construyamos un futuro sostenible. Diálogos de supervivencia. Madrid: Cambridge UniversityPresss. Capítulo 9.

Vilches, A. y Gil Pérez, D. (2013). La Ciencia de la Sostenibilidad en la formación del profesorado de ciencias, Revista Eureka sobre Enseñanza y Divulgación de las Ciencias,10 (Número Extraordinario), pp. 749-762. Recuperado de: http://hdl.handle.net/10498/15625 http://reuredc.uca.es

Vilches, A. y Gil-Pérez, D. (2016). La transición a la Sostenibilidad como objetivo urgente para la superación de la crisis sistémica actual. Revista Eureka sobre Enseñanza y Divulgación de las Ciencias, 13 (2), 395-407. Recuperado de: http://hdl.handle.net/10498/18296 http://reuredc.uca.es

Vilches, A., Macías, O. y Gil-Pérez, D. (2014). La transición a la Sostenibilidad. Un desafío urgente para la ciencia, la educación y la acción ciudadana. Temas clave de reflexión y acción. Madrid: OEI. ISBN 978-84-7666-204-5. 